\title{
GENERALIZED ADAPTIVE VECTOR SIGMA FILTERS
}

\author{
R. Lukac ${ }^{1}$, B. Smolka ${ }^{2}$, K.N. Plataniotis ${ }^{3}$ and A.N. Venetsanopoulos ${ }^{3}$ \\ ${ }^{1}$ Slovak Image Processing Center, Jarkova 343, \\ 04925 Dobsina, Slovak Republic, lukacr@ieee.org \\ ${ }^{2}$ Department of Automatic Control, Silesian University. of Technology, \\ Akademicka 16 Str., 44-101 Gliwice, Poland, bsmolka@ia.polsl.gliwice.pl \\ ${ }^{3}$ Edward S. Rogers Sr. Department of Electrical and Computer Engineering, University of Toronto, \\ 10 King's College Road, Toronto, Canada, \{kostas, anv $\}$ dsp.toronto.edu
}

\begin{abstract}
In this paper we provide a new filtering scheme for the detection and the removal of impulsive noise in digital color images. The proposed adaptive nonlinear vector filters take the advantages of the robust order-statistic theory, generalized directional distance filter and standard sigma filter concept. The principles of the design are explained in detail. Simulation studies indicate that the proposed method is computationally attractive and is able to achieve excellent balance between the image-detail preservation and the noise attenuation.
\end{abstract}

\section{INTRODUCTION}

In the field of color image processing, it has been widely recognized that the processing of color image data as vector fields is desirable due to the high correlation that exists between the image channels, and that the nonlinear vector processing of color images is the most effective way to filter out noise. In this way, a number of reference vector filters such as vector median filter (VMF) [1], basic vector directional filter (BVDF) [11] and the directional distance filter (DDF) [2] was developed. These nonlinear filters, based on the ordering operation [7-10], provide the lowest ranked vector as robust estimation in environments corrupted by bit errors, impulsive noise and outliers.

Because impulsive noise affects a certain number of samples, whereas other samples remain unchanged, ideally is to divide the samples into two classes such as a class of noisy samples and a class of desired (noise-free) samples. Then, the filter is applied only to noisy samples and preserves the uncorrupted image information [4],[5]. Note that the processing precision depends on the detection accuracy and also on the estimation capability of chosen filtering scheme. Usually, the robust behaviour is provided by nonlinear filters based on the order-statistic theory [6].

\section{RELEVANT VECTOR FILTERING SCHEMES}

Let $y(x): Z^{\prime} \rightarrow Z^{m}$ represent a multichannel image, where $l$ is an image dimension and $m$ characterizes a number of channels $(m=3$ for color images). Let $W=\left\{\mathbf{x}_{i} \in Z^{\prime} ; i=1,2 \ldots, N\right\}$ represent a filter window of a finite size $N$, where $\mathbf{x}_{1}, \mathbf{x}_{2}, \ldots, \mathbf{x}_{N}$ is a set of noisy samples. Note that the position of the filter window is determined by the central sample $\mathbf{x}_{(N+1) / 2}$.

In general, the difference between two multichannel samples $\mathbf{x}_{i}=\left(x_{i 1}, x_{i 2}, \ldots, x_{i m}\right)$ and $\mathbf{x}_{j}=\left(x_{j 1}, x_{j 2}, \ldots, x_{j m}\right)$ can be quantified through the commonly used Minkowski metrics [8]:

$$
\left\|\mathbf{x}_{i}-\mathbf{x}_{j}\right\|_{\gamma}=\left(\sum_{k=1}^{m}\left|x_{i k}-x_{j k}\right|^{\gamma}\right)^{\frac{1}{\gamma}}
$$

where $\gamma$ characterizes the specific kind of used norm, $m$ is the dimension (number of channels) of vectors and $x_{i k}$ is the $k$ th element of the sample $\mathbf{x}_{i}$. Note that the wellknown Euclidean distance is obtained for $\gamma=2$.

Let us consider the input sample $\mathbf{x}_{i}, i=1,2, \ldots, N$, associated with the distance measure $L_{i}[1],[8]$ given by

$$
L_{i}=\sum_{j=1}^{N}\left\|\mathbf{x}_{i}-\mathbf{x}_{j}\right\|_{\gamma} \quad \text { for } i=1,2, \ldots, N
$$

Another way [4],[8],[11] of determining the distance between multichannel samples is based on the angle between two multichannel vector samples $\mathbf{x}_{i}=\left(x_{i 1}, x_{i 2}, \ldots, x_{i m}\right)$ and $\mathbf{x}_{j}=\left(x_{j 1}, x_{j 2}, \ldots, x_{j m}\right)$ :

$$
\mathrm{A}\left(\mathbf{x}_{i}, \mathbf{x}_{j}\right)=\cos ^{-1}\left(\frac{\mathbf{x}_{i} \cdot \mathbf{x}_{j}^{T}}{\left|\mathbf{x}_{i}\right| \cdot\left|\mathbf{x}_{j}\right|}\right)
$$

Let each input sample $\mathbf{x}_{i}$, for $i=1,2, \ldots, N$, be also associated with the aggregated angular distance defined by

$$
\alpha_{i}=\sum_{j=1}^{N} \mathrm{~A}\left(\mathbf{x}_{i}, \mathbf{x}_{j}\right) \text { for } i=1,2, \ldots, N
$$

If the ordering criterion is expressed through products 


$$
\begin{gathered}
\Omega_{i}=L_{i}^{1-p} \cdot \alpha_{i}^{p} \text { for } i=1,2, \ldots, N \\
\Omega_{i}=\left(\sum_{j=1}^{N}\left\|\mathbf{x}_{i}-\mathbf{x}_{j}\right\|_{\gamma}\right)^{1-p} \cdot\left(\sum_{j=1}^{N} \mathrm{~A}\left(\mathbf{x}_{i}, \mathbf{x}_{j}\right)\right)^{p} \text { for } i=1,2, \ldots, N
\end{gathered}
$$

where $p \in\langle 0,1\rangle$ is a power parameter; and the ordered set is given by

$$
\Omega_{(1)} \leq \Omega_{(2)} \leq \ldots \leq \Omega_{(N)}
$$

then the same ordering scheme applied to the input set results in the ordered sequence

$$
\mathbf{x}^{(1)} \leq \mathbf{x}^{(2)} \leq \ldots \leq \mathbf{x}^{(N)}
$$

The sample $x^{(1)}$ associated with $\Omega_{(i)}$ represents the output of the Directional Distance Filter (DDF) [2]. If $p=0$, the DDF operates as the Vector Median Filter (VMF) [1], whereas for $p=1$, the DDF is equivalent to the Basic Vector Directional Filter (BVDF) [11].

\section{PROPOSED METHOD}

The above-described well-known filtering schemes such as VMF, BVDF and DDF are designed to perform the fixed amount of smoothing. In many applications, it may become an undesired property because the used filter will introduce too much smoothing resulting in a blurring of image edges and thin details. Note that the introduced blurring may be more objectionable than original noise.

For that reason, the common problem is how to preserve some desired signal features, when removing efficiently the noise elements. Ideally, the noise reduction filter should be designed in such a way that the noise-free samples should be invariant to the filtering operation and only noise-corrupted pixels should be affected by the filter action. In order to provide the adaptive trade-off between the identity filter and the smoothing function and still keep the directional distance filter structure, we present a new filtering scheme, which makes use of statistical operators such as mean and standard deviation of multichannel samples spanned by the filtering window.

The proposed filter is based on the concept of sigmafilter proposed by Lee [3]. Let us consider the statistical measure called standard deviation, generally written as

$$
\sigma=\sqrt{\frac{1}{N} \sum_{i=1}^{N}\left(x_{i}-\mu\right)^{2}}
$$

where $\mu$ is a mean of the observed data $x_{1}, x_{2}, \ldots, x_{N}$. If the definition (9) is rewritten for a vector case and it is applied to the input set $\mathbf{x}_{1}, \mathbf{x}_{2}, \ldots, \mathbf{x}_{N}$, then [5]:

$$
\sigma_{\gamma}=\sqrt{\frac{1}{N} \sum_{i=1}^{N}\left(\left\|\mathbf{x}_{i}-\mu\right\|_{\gamma}\right)^{2}}
$$

where $N$ is the window size, $\gamma$ denotes the chosen norm generally, and $\boldsymbol{\mu}=\left(\mu_{1}, \mu_{2}, \ldots, \mu_{m}\right)$ is the sample mean.
The angular definition of the standard deviation is given by

$$
\sigma_{A}=\sqrt{\frac{1}{N} \sum_{i=1}^{N} A^{2}\left(\mathbf{x}_{i}, \mu\right)}
$$

In terms of the DDF filter structure, the combination of $\sigma_{\gamma}$ and $\sigma_{A}$ is expressed as follows:

$$
\begin{gathered}
\sigma_{\gamma A}=\left(\sigma_{\gamma}\right)^{1-p} \cdot\left(\sigma_{A}\right)^{p} \\
\sigma_{\gamma A}=\sqrt{\left(\frac{1}{N^{2}} \sum_{i=1}^{N}\left(\left\|\mathbf{x}_{i}-\mu\right\|_{\gamma}\right)^{2}\right)^{1-p}\left(\sum_{i=1}^{N} A^{2}\left(\mathbf{x}_{i}, \mu\right)\right)^{p}}
\end{gathered}
$$

where $p$ is the same power parameter as in the DDF structure.

Let us compute the distance $\zeta_{\gamma}$ between the central sample $\mathbf{x}_{(N+1) / 2}$ and the sample mean $\mu$ such that

$$
\zeta_{\gamma}=\left\|\mathbf{x}_{(N+1) / 2}-\boldsymbol{\mu}\right\|_{\gamma}
$$

and also the angle $\varsigma_{\gamma}$ between $\mathbf{x}_{(N+1) / 2}$ and $\boldsymbol{\mu}$ as follows:

$$
\varsigma_{A}=A\left(\mathbf{x}_{(N+1) / 2}, \boldsymbol{\mu}\right)
$$

Then, we can determine the difference between $\mathbf{x}_{(N+1) / 2}$ and $\boldsymbol{\mu}$ through the combined distance given by

$$
\begin{gathered}
\varsigma_{\gamma A}=\left(\zeta_{\gamma}\right)^{1-p} \cdot\left(\zeta_{A}\right)^{p} \\
\zeta_{Y A}=\left(\left\|\mathbf{x}_{(N+1) / 2}-\mu\right\| \|_{\gamma}^{1-p} \cdot\left(A\left(\mathbf{x}_{(N+1) / 2}, \boldsymbol{\mu}\right)\right)^{p}\right.
\end{gathered}
$$

Thus, the corruption of the central sample $\mathbf{x}_{(N+1) / 2}$ is determined by a simple comparison of the standard deviation $\sigma_{\gamma A}$ and the product $\zeta_{\gamma A}$. This comparison forms the following inequality $\zeta_{\gamma A} \geq \sigma_{\gamma A}$.

If the combined distance $\zeta_{\gamma A}$ is greater than or equal to the combined standard deviation $\sigma_{\gamma A}$, then the central sample $\mathbf{x}_{(N+1) / 2}$ is probably noisy because the corresponding combined distance $\zeta_{\gamma,}$ reflects the significant difference between $\mathbf{x}_{(N+1) / 2}$ and its neighborhoods. The high similarity between $\mathbf{x}_{(N+1) / 2}$ and the input set $\mathbf{x}_{1}, \mathbf{x}_{2}, \ldots, \mathbf{x}_{N}$ is reflected by $\zeta_{y A}<\sigma_{\gamma A}$.

When the above-mentioned detection rule is introduced to the DDF filter structure, the output $y$ of the proposed adaptive DDF sigma filter is given by

$$
\begin{aligned}
& \text { IF } \varsigma_{\gamma A} \geq \sigma_{\gamma A} \text { THEN } \mathbf{y}=\mathbf{x}^{(1)} \\
& \text { ELSE } \mathbf{y}=\mathbf{x}_{(N+1) / 2}
\end{aligned}
$$

where $x^{(1)}$ characterizes the DDF output (8) and $\mathbf{x}_{(N+1) / 2}$ is the input central sample.

It is clear. that the standard DDF operation is performed for satisfied condition $\zeta_{\gamma A} \geq \sigma_{\gamma A}$, i.e. the effect of the DDF filtering is restricted only to noisy samples. If $\zeta_{\gamma A}<\sigma_{\gamma A}$, then $\mathbf{x}_{(N+1) / 2}$ has similar properties as the other input samples which indicates that it is the most probably noise-free and no filtering operation is performed. 


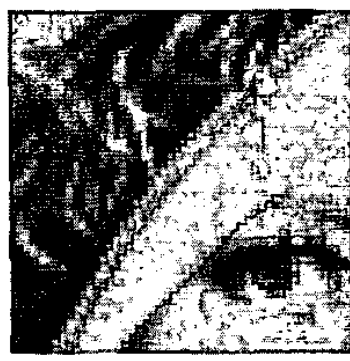

(a)

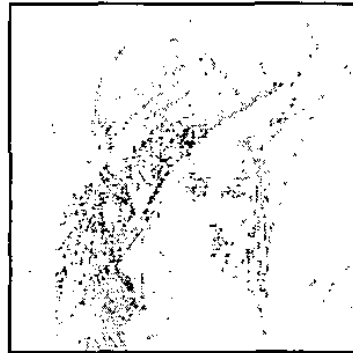

(e)

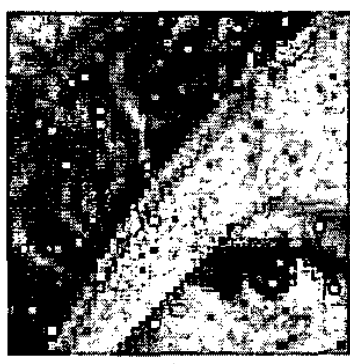

(b)

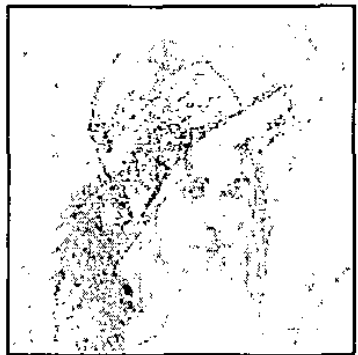

(f)

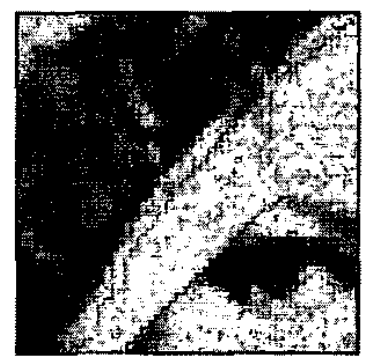

(c)

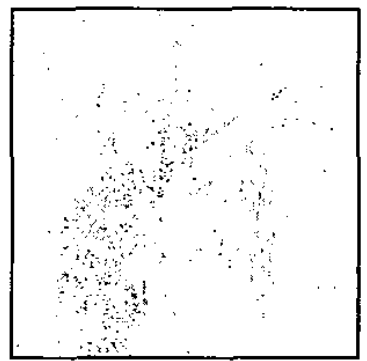

(g)

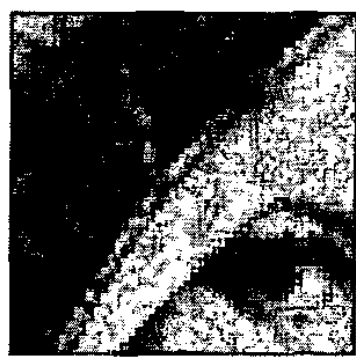

(d)

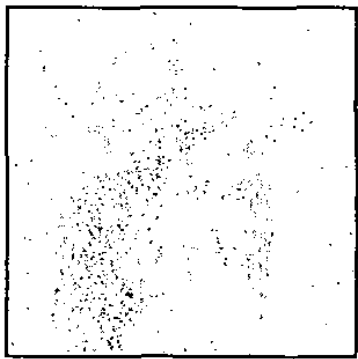

(h)

Fig. 1 Achieved results using the test image Lena, (a-d) Zoomed results: (a) original image, (b) original corrupted by $5 \%$ impulsive noise, (c) VMF output, (d) SVMF output; (e-h) Estimation errors: (e) VMF, (f) DDF, (g) SVMF, (h) SDDF

\section{EXPERIMENTAL RESULTS}

We used the well-known test color images Lena and Peppers that have been corrupted by impulsive noise [9]. To evaluate the achieved results, objective criteria [8] such as Mean Absolute Error (MAE), Mean Square Error (MSE) and Normalized Color Difference (NCD), that reflect the signal-detail preservation, the noise suppression and color chromaticity preservation, have been used.

The results shown in Fig. 1 show the excellent detail preservation capability of the proposed method. It can be seen that the sigma VMF (SVMF) excellently preserves image edges and thin details (Fig.1d) and its output is comparable with the original image (Fig.la), whereas standard VMF blurs the output image (Fig.1c). The same behavior can be observed from results shown in Fig.le-f, where the performance of standard filtering schemes (Figl.e,f) is accompanied with significant estimation error. In the case of the proposed sigma DDF (SDDF) and its special cases such as SVMF and sigma BVDF (SBVDF), the estimation error is significantly reduced. Note that the performance of sigma DDF filters decreases (Fig.2) with the increased power parameter $p$.

The robustness of the proposed methods is confirmed by the results shown in Fig.3, where the dependence of the filter performance on the degree of the noise corruption is shown. It can be observed again, that the proposed SVMF, SBVDF and SDDF achieve better results than standard filtering schemes, especially in terms of MAE and NCD criteria. However, in terms of MSE criteria, their performance can fail with a large amount of injected noise.
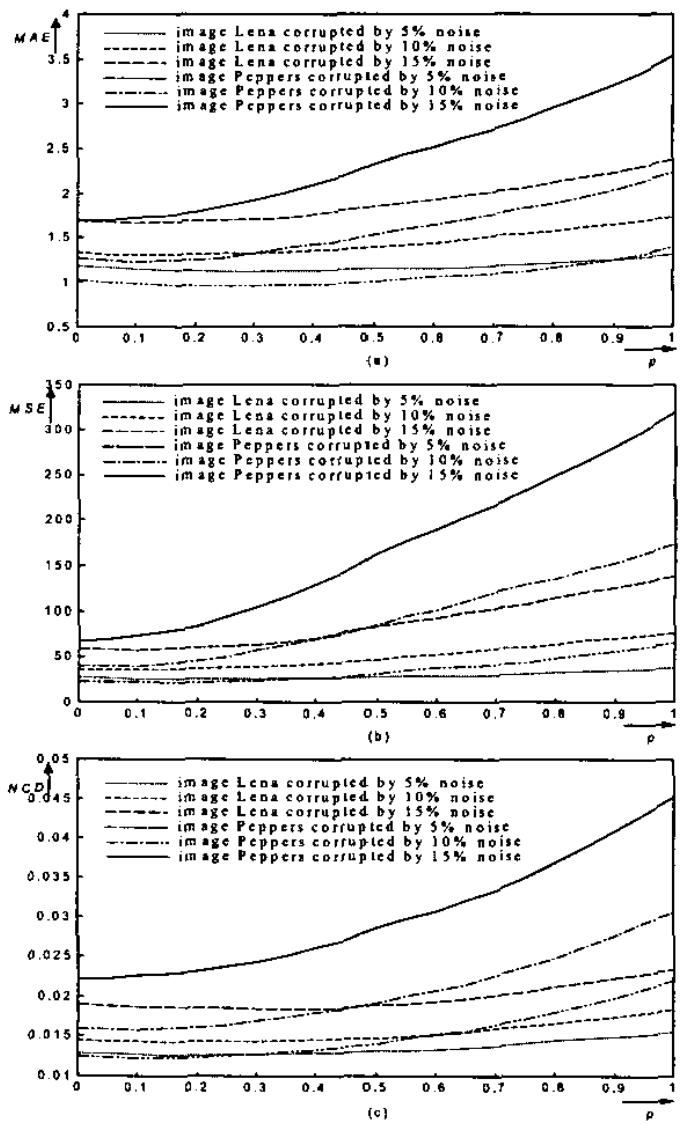

Fig. 2 Objective measures of the proposed method in dependence on the power parameter p. (a) MAE, (b) MSE, (c) NCD 

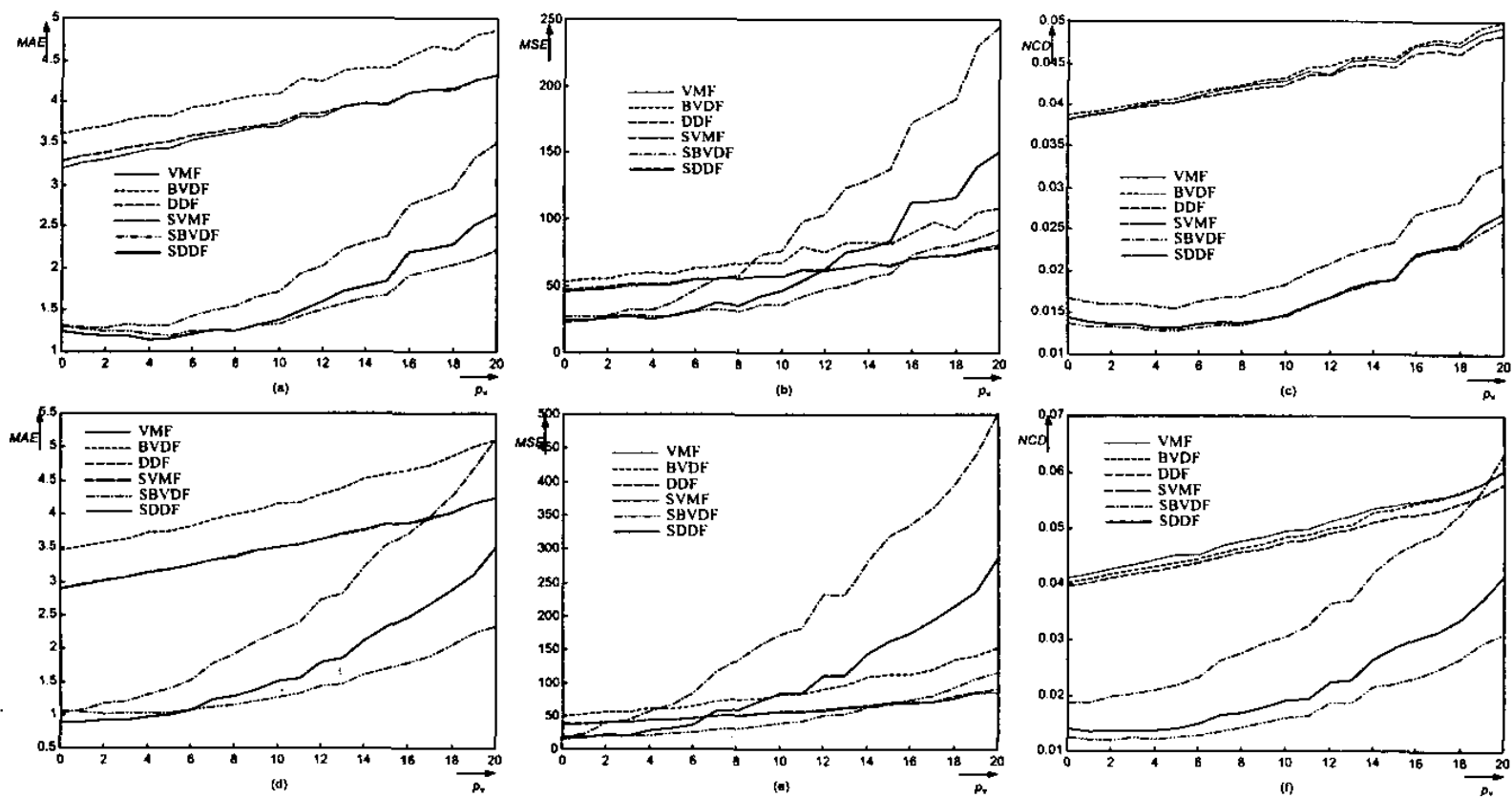

Fig. 3 Objective measures of the relevant methods in dependence on the noise corruption

Table 1 Performance of methods related to the image Lena.

\begin{tabular}{|c|r|r|r|r|r|r|}
\hline Image & \multicolumn{3}{|c|}{$5 \%$} & \multicolumn{3}{c|}{$10 \%$} \\
\hline Method & MAE & MSE & NCD & MAE & MSE & NCD \\
\hline Noisy & 3.762 & 427.3 & 0.045 & 7.312 & 832.0 & 0.084 \\
\hline VMF & 3.430 & 50.8 & 0.040 & 3.687 & 56.5 & 0.043 \\
\hline BVDF & 3.818 & 58.6 & 0.041 & 4.099 & 67.6 & 0.043 \\
\hline DDF & 3.509 & 52.3 & 0.040 & 3.733 & 57.3 & 0.042 \\
\hline SVMF & 1.192 & 27.3 & 0.013 & 1.332 & 36.2 & 0.015 \\
\hline SBVDF & 1.313 & 37.6 & 0.016 & 1.721 & 76.3 & 0.018 \\
\hline SDDF & 1.153 & 28.2 & 0.013 & 1.378 & 46.7 & 0.015 \\
\hline
\end{tabular}

Table 2 Performance of methods related to the image Peppers.

\begin{tabular}{|c|r|r|r|r|r|r|}
\hline Image & \multicolumn{3}{|c|}{$5 \%$} & \multicolumn{3}{c|}{$10 \%$} \\
\hline Method & MAE & MSE & NCD & MAE & MSE & NCD \\
\hline Noisy & 3.988 & 486.1 & 0.044 & 7.677 & 943.3 & 0.087 \\
\hline VMF & 3.169 & 43.9 & 0.045 & 3.503 & 55.0 & 0.049 \\
\hline BVDF & 3.740 & 60.7 & 0.044 & 4.151 & 82.7 & 0.048 \\
\hline DDF & 3.182 & 44.6 & 0.043 & 3.512 & 56.6 & 0.048 \\
\hline SVMF & 1.033 & 23.0 & 0.012 & 1.265 & 39.9 & 0.016 \\
\hline SBVDF & 1.391 & 65.9 & 0.022 & 2.239 & 173.4 & 0.031 \\
\hline SDDF & 1.004 & 31.1 & 0.014 & 1.514 & 84.6 & 0.019 \\
\hline
\end{tabular}

\section{CONCLUSION}

In this paper, a generalized adaptive DDF sigma filter for color image filtering has been proposed. The results show excellent detection and signal-detail preservation capabilities of the new approach, while the impulsive noise attenuation characteristics of standard DDF filter are still held. Clearly, the new filter outperforms the standard vector filtering schemes. In general, the best results were achieved by adaptive sigma VMF filter that is the most computational attractive case of the proposed method.

[1] J. Astola, P. Haavisto, and Y. Neuvo, "Vector Median Filters," Proc. IEEE, Vol.78, No.4, pp.678-689, April 1990.

[2] D.G. Karakos, and P.E. Trahanias, "Generalized Multichannel Image-Filtering Structure," IEEE Transactions on Image Processing, Vol.6, No.7, pp.1038-1045, July 1997.

[3] J.S. Lee, "Digital Image Smoothing and the Sigma Filter," Comp. Vision, Graphics, Image Processing, Vol. 24, No.2, pp.255-269, Nov. 1983.

[4] R. Lukac, "Color Image Filtering by Vector Directional Order-Statistics," Pattern Recognition and Image Analysis, Vol. 12, No. 3, pp. 279-285, August 2002.

[5] R. Lukac, B. Smolka and K. N. Plataniotis, "Color Sigma Filter," Proc. IWSSIP'02 in Manchester, UK, pp.559-565, 2002.

[6] I. Pitas, and A.N. Venetsanopoulos, "Order Statistics in Digital Image Processing," Proceedings of the IEEE, Vol.80, No.12, pp.1892-1919, December 1992.

[7] K.N. Plataniotis, D. Androutsos, V. Sri and A.N. Venetsanopoulos, "A Nearest Neighbour Multichannel Filter," Electronic Letters, Vol. 31, pp. 1910-1911, 1995.

[8] K.N. Plataniotis, and A.N. Venetsanopoulos, Color Image Processing and Applications, Springer Verlag, 2000.

[9] B. Smolka, A. Chydzinski, K. Wojciechowski, K.N. Plataniotis and A.N. Venetsanopoulos, "On the Reduction of Impulsive Noise in Multichannel Image Processing," Optical Engineering, Vol.40, pp.902-908, 2001.

[10] K. Tang, J. Astola, and Y. Neuvo, "Nonlinear Multivariate Image Filtering Techniques," IEEE Trans. Image Processing, Vol.4, No.6, pp.788-798, June 1995.

[11] P.E. Trahanias, D. Karakos, and A.N. Venetsanopoulos, "Directional Processing of Color Images: Theory and Experimental Results," IEEE Trans. Image Processing, Vol.5, No.6, pp.868-881, June 1996. 